\title{
LA INTERPRETACIÓN DE TEXTOS EN LOS COMIENZOS DE LA ALFABETIZACIÓN DEL NIÑO SORDO
}

\section{TEXT INTERPRETATION BY DEAF CHILDREN DURING EARLY LITERACY ACQUISITION}

Celia Zamudio Mesa

Escuela Nacional de Antropología e Historia, Licenciatura en Lingüística

Rodrigo Ortiz Sánchez

Escuela Nacional de Antropología e Historia, Licenciatura en Lingüística

RESUMEN

El objetivo del estudio es indagar si los niños sordos que están aprendiendo a leer siguen una evolución semejante a la de la lectura prealfabética de los oyentes, según los datos de la investigación psicogenética de la escritura (Ferreiro \& Teberosky, 1979). La situación experimental involucró la interpretación en lengua de señas mexicana (LSM) de enunciados escritos en español: los niños recibían del entrevistador la lectura de los enunciados; después debían decidir qué estaba escrito y señalar la parte de texto donde pensaban que se encontraba. Participaron 22 niños sordos profundos (15 hombres y 7 mujeres con un rango de edad entre 6 y 9 años) de una escuela primaria privada de la Ciudad de México especializada en la enseñanza de LSM. Los resultados muestran cómo la reconstrucción de lo escrito que realizan los niños sordos es también un proceso evolutivo, aunque con diferencias importantes respecto de los oyentes, debidas principalmente a la estructura de las lenguas involucradas. El estudio discute tales discrepancias y aporta datos que permiten reflexionar sobre las maneras como se alfabetiza a los sordos.

Palabras Clave: lectura prealfabética; psicogénesis del lenguaje escrito; adquisición del español

Estudios de Lingüística Aplicada, año 35, número 66, diciembre de 2017, pp. 77-107 doi: 10.22201/enallt.01852647p.2017.66.829 
Abstract

This research investigates if deaf children who are learning to read follow the same patterns as hearing children, according to the psychogenetic theory of writing acquisition (Ferreiro \& Teberosky, 1979). The experimental design involved reading three sentences written in Spanish using Mexican sign language (LSM). The interviewer presented the interpretation and, afterwards, the children had to identify the elements from the passage that were written and where in the text these were located. This study involved the participation of 22 deaf children (15 boys and 7 girls aged from 6 to 9 years old) that were studying in a private school in Mexico City specialized in LSM, reading and writing techniques for deaf people. The results show how the reconstruction of what was written made by the deaf children is also a developing process, although with major differences relative to the results obtained from hearing children. This is mainly due to the structure of the languages involved. The study discusses such differences and introduces new data that allow us to reflect on ways to teach reading and writing skills to deaf people.

KEYWORDS: prealphabetic reading; psychogenetic theory; reading development; Spanish acquisition

Fecha de recepción: 1 de agosto de 2016

Fecha de recepción de la versión revisada: 19 de junio de 2017

Fecha de aceptación: 20 de junio de 2017

La correspondencia relacionada con este artículo debe dirigirse a:

Celia Zamudio Mesa

celyaz@prodigy.net.mx

Estudios de Lingüística Aplicada, año 35, número 66, diciembre de 2017, pp. 77-107

doi: 10.22201/enallt.01852647p.2017.66.829 


\section{INTRODUCCIÓN}

Numerosas investigaciones sobre el desarrollo de la alfabetización o literacidad de los niños sordos destacan la escasa comprensión de la lengua escrita que logran al término de la educación básica. La población escolar sorda de los países angloparlantes no alcanza el nivel de lectura correspondiente a su edad y grado escolar (Marschark, 1997; Musselman, 2000); lo mismo se reporta para los países hispanohablantes (Figueroa \& Lissi, 2005; Alegría \& Domínguez, 2009; Massone, Buscaglia \& Bogado, 2010). Pietrosemoli (2007) menciona además que, de acuerdo con las estadísticas de la Universidad Gallaudet, la mayoría de los estudiantes sordos que ingresa a dicha institución no está capacitada para leer los libros de texto; asimismo, la mayor parte de los adultos sordos no está en condiciones de utilizar la lectura como una herramienta de trabajo.

Entre los problemas de los sordos para alcanzar el dominio de la lengua escrita se ha señalado su escaso vocabulario y la dificultad que presentan para entender y utilizar las palabras funcionales y la morfología de la lengua escrita, como las concordancias de género o número (Fabbretti, 1997; Niederberger, 2007; Herrera, Puente \& Alvarado, 2014). Tales limitaciones se han atribuido, en el caso de los hipoacúsicos, a un insuficiente dominio de la lengua oral (Alegría \& Domínguez, 2009), y en los sordos profundos, de la lengua de señas (Goldin Meadow \& Mayberry, 2001). Lo contrario, esto es, la correlación positiva entre el dominio de la lengua de señas y un buen desempeño lector también se ha mostrado (Hoffmeister, 2000; Strong \& Prinz, 2000).

Ahora bien, a pesar de que se reconoce la diferencia estructural entre las lenguas escrita y de señas y se argumente en favor de una educación bilingüe para la población sorda, poco se ha planteado en estas discusiones el carácter puramente visual que tiene la segunda lengua para los sordos, así como la posibilidad de aprender la morfología y sintaxis de la segunda lengua por dicha vía. Excepciones relevantes son las reflexiones de Musselman (2000) y Paul (2003) acerca del aprendizaje del inglés escrito, las realizadas por Niederberger (2007) y Burgat (2009) sobre el francés y las de Herrera, Puente y Alvarado (2014) en torno del español.

La escasa preocupación sobre el fundamento visual de la lengua escrita en el proceso de adquisición de los niños sordos se entiende cuando se examina la 
concepción de escritura detrás de estos estudios. El principio dominante es que la escritura alfabética transcribe los sonidos del lenguaje oral, y es sobre esta relación que un grupo importante de investigadores ha centrado el proceso de aprendizaje de los sordos: Leybaert (1998), Leybaert, Content y Alegría (2008) y Alegría y Domínguez (2009), entre otros. Aunque no se conoce claramente cómo los niños sordos organizan la información fonológica de la lengua hablada ni en qué medida esto constituye un factor crítico para el desarrollo de la lectura y escritura (Allman, 2002; Miller \& Clark, 2011), los autores que sostienen esta perspectiva insisten en la necesidad de desarrollar en los sordos la conciencia fonológica. Para ello se ha diseñado una combinación de estrategias que incluye la lectura labial, el Cued Speech (CS: un conjunto de señas que enfatizan las posiciones articulatorias), el deletreo manual, la fonoarticulación y, desde luego, la exposición a la escritura (Leybaert \& Alegría, 1995; Alegría \& Lechat, 2005; Herrera, Puente, Alvarado \& Ardila, 2007).

Una variante de esa misma idea consiste en pensar la escritura como un sistema que establece correspondencias inmediatas con los elementos de una lengua oral (mapping es el término utilizado en inglés para referirse a este fenómeno). Consecuencia de ello es la invención de varios sistemas del grupo conocido como Manually Coded English (MCE), los cuales toman prestados signos del sistema American Sign Language (ASL) y de la estructura sintáctica del inglés con el propósito de enseñar esta lengua no solamente mediante la lectura de labios del inglés hablado, sino también de los patrones manuales creados especialmente para signarlo (Goldin Meadow \& Mayberry, 2001).

Este estudio parte de una noción distinta de la escritura, construida con base en los datos de la investigación psicogenética de la lengua escrita. Desde esta perspectiva, la lengua escrita se concibe como un sistema de representación, y no un código de transcripción (Ferreiro, 1998). La diferencia entre uno y otro, afirma Ferreiro (1998: 15), es que en los códigos de transcripción tanto los elementos como sus relaciones están predeterminados, como en el código morse o el código binario computacional, que toman una representación ya constituida y crean nuevas marcas que relacionan con las anteriores. En cambio, en los sistemas de representación ni los elementos ni las relaciones que los componen están predeterminados; por eso, en el proceso histórico de invención de las es- 
crituras los aspectos del lenguaje reconstruidos en la representación gráfica han sido muy variados.

La noción de escritura planteada desde la psicogénesis se opone a la sostenida en la tradición escolar, lingüística y filosófica: el ideal de que la escritura, especialmente, la alfabética es una reproducción, registro o copia de elementos lingüísticos presentes en la cognición humana. Esta hipótesis se apoya en la idea, también hipotética, de que los elementos y unidades lingüísticas son un objeto primario, es decir, representaciones genéticamente anteriores a la escritura e independientes de la materia en que se presenta. Si este fuera el caso, cabría explicar, por un lado, por qué las didácticas asociacionistas no son suficientes para estimular y completar el proceso de adquisición en los oyentes y, por otro, el hecho de que la conciencia de los fonemas no aparezca antes de los cinco o seis años de edad; y que, por el contrario, se desarrolle a medida que los humanos logran el dominio de la escritura alfabética (Morais \& Kolinsky, 1995; Vernon \& Ferreiro, 1999).

La teoría psicogenética trata la escritura como un objeto de conocimiento y al sujeto del aprendizaje como sujeto cognoscente, es decir, "un sujeto que trata activamente de comprender el mundo que lo rodea, y de resolver sus interrogantes para comprenderlo" (Ferreiro \& Teberosky, 1979: 28). El sujeto de la teoría piagetiana organiza y reorganiza las propiedades de los objetos que va logrando aislar, mediante la acción efectiva y la acción interiorizada del pensamiento. No espera a que el conocimiento le sea enseñado.

Otro principio fundamental de la teoría piagetiana es que los estímulos no inciden directamente sobre el sujeto; primero son transformados por los esquemas de conocimiento y, gracias a esta transformación, es que el sujeto puede brindar una interpretación del estímulo. Ningún estímulo es el mismo a menos que los esquemas asimiladores disponibles también lo sean (Ferreiro \& Teberosky, 1979: 30). Por esta razón es que ningún aprendizaje parte de un punto de vista absoluto: "por nuevo que sea el contenido a conocer, este deberá necesariamente ser asimilado por el sujeto y, según los esquemas asimiladores a disposición, la asimilación será más o menos deformante", explican Ferreiro y Teberosky (1979: 33). Así, puesto que el objeto a ser abordado solo está disponible en la medida en que el sujeto posee los esquemas necesarios para entenderlo, el 
sujeto es la pieza central durante el proceso de aprendizaje y no el objeto, ni la persona que enseña ni cómo lo hace.

Al ser un objeto que circula intensamente, sobre todo en los contextos urbanos, la escritura no es ajena a los niños. Ellos no esperan hasta su ingreso a la escuela para aprender sobre la lengua escrita; por el contrario, logran avances importantes en la comprensión de la naturaleza del sistema incluso antes de haber recibido información sistemática en la escuela (Teberosky, 1989). Todos los niños, aun aquellos hijos de analfabetas, desarrollan ideas acerca de la escritura a partir de la interacción con ella.

El proceso que siguen para entender la lengua escrita es muy semejante al de su construcción histórica, debido a que ni los elementos ni las relaciones del sistema lingüístico que entran en la representación gráfica les son asequibles de inmediato. De acuerdo con Ferreiro (1998: 15), los niños re-inventan el sistema porque, "para poder utilizar estos elementos como elementos de un sistema, deben comprender su proceso de construcción y sus reglas de producción”. De ahí que los niños establezcan en un principio que la escritura representa los nombres de las cosas y utilicen las letras únicamente para crear diferencias entre ellos; o que, posteriormente, cuando les otorgan valor sonoro, sea silábico y no segmental.

Tomando entonces como punto de partida la idea de que el niño reconstruye el sistema de representación, este trabajo se propone indagar el proceso de reconstrucción de lo escrito de los niños sordos, a partir de un acto de lectura que consiste en 1) proporcionar al niño la interpretación en lengua de señas mexicana (LSM) de un enunciado escrito en español y 2) pedirle que identifique qué puede estar escrito y dónde pueden estar escritas las partes que lo componen. El estudio forma parte de un trabajo de tesis de licenciatura que incluyó otras situaciones del protocolo experimental propuesto por Ferreiro y Teberosky (1979) para investigar el proceso de adquisición de la lengua escrita.

\subsection{La teoría psicogenética de la lectura prealfabética del oyente}

Desde la perspectiva psicogenética, el que un niño no pueda leer en el sentido convencional que tenemos de la lectura no es indicio de que no conozca nada sobre los textos escritos. Mucho antes de recibir instrucción formal sobre la lectura, los niños oyentes pueden reconocer los objetos con texto y asignarles la 
función "para leer". Igualmente, pueden imitar las acciones de los adultos relacionadas con los libros como sostenerlos, mirarlos, hojearlos o enunciar algo de manera plausible mientras los miran: "el texto es visto como portador de algún contenido, sugiere algo; las preguntas ‘¿qué dice?’ o ‘¿dice algo?’, son aceptadas como pertinentes frente a un texto" (Ferreiro \& Teberosky, 1979: 82).

Alrededor de los cuatro años, los niños comienzan a distinguir entre imágenes y texto. Esta distinción se evidencia cuando afirman frente al dibujo: "esto es un...", mientras que ante el texto mencionan el nombre del objeto dibujado o dicen explícitamente: "aquí dice..." (Ferreiro \& Teberosky, 1979: 88). El dúo imagen-texto parece funcionar en un inicio como una unidad que expresa el sentido del mensaje gráfico: de un lado está la representación del objeto y del otro la de su nombre (Ferreiro \& Teberosky, 1979). Sin embargo, la relación se pone en conflicto cuando no hay correspondencia entre las partes del enunciado escrito y los elementos de la imagen. Los casos en que hay una sola palabra, por ejemplo: granja o corral, pero más de un elemento en el dibujo (varios animales de la granja dentro de un corral), o en que son varias las palabras: el mango está maduro, pero un solo elemento en el dibujo (un mango), son difíciles de resolver para el niño prealfabetizado porque no puede ajustar las palabras del texto con los nombres de las figuras que aparecen en la imagen.

Los problemas de interpretación son distintos cuando se trata de textos que carecen de imágenes. Previo a la alfabetización, los niños pueden reconocer globalmente un enunciado que les es leído; pueden repetirlo palabra por palabra y señalarlo desde el principio hasta el final, al igual que el lector lo presenta. Pero, cuando tienen que relacionar las partes del texto con las del enunciado oral proporcionado por el lector, no pueden asignar las palabras del enunciado oral a las del escrito.

Una de las dificultades para el niño oyente es cómo dividir la emisión y hacer corresponder las partes encontradas con las del texto, puesto que en la enunciación oral no están presentes las separaciones que se indican en la escritura (Ferreiro \& Teberosky, 1979). La otra consiste en aceptar que todo lo que se enuncia esté también escrito y en el mismo orden. Admitir la posibilidad de que el enunciado se lea en lo escrito no significa para el niño que todo se escriba. Las expectativas del niño revelan una concepción de la escritura diferente a la del adulto alfabetizado: "el texto sirve para provocar o sugerir una emisión oral, pero no la determina totalmente" (Ferreiro \& Teberosky, 1979: 145). 
Puesto que la hipótesis inicial del niño sobre lo escrito es que se representan solamente los nombres, le resulta difícil admitir que pueden estar escritos otros elementos del enunciado oral (Ferreiro \& Teberosky, 1979). El reconocimiento de que todo lo dicho debe estar escrito (por ejemplo, los verbos y, sobre todo, los elementos funcionales) forma parte de un proceso evolutivo de la lectura prealfabética que implica analizar el enunciado en sus partes componentes.

Ferreiro y Teberosky (1979) estudiaron dicho proceso de desarrollo mediante una situación experimental que consistía en escribir delante del niño un enunciado que después le era leído, mientras se lo señalaba de corrido con el dedo. Las oraciones presentadas fueron las siguientes: Papá patea la pelota, la nena come/compró un caramelo, el perro corrió al gato y elosocomemiel ( $\mathrm{sin}$ espacios). Una vez leído el enunciado se le preguntaba al niño dónde pensaba que se encontraban las palabras que lo integraban. Por ejemplo: ¿Dónde escribí papá? ¿Dónde escribí pelota? Y así con cada una de ellas, comenzando siempre con un sustantivo y variando el orden de las preguntas. También se podía señalar una parte del texto y preguntar por lo que estaba escrito allí.

A partir de las partes del enunciado oral que los niños identificaban y la localización que hacían de ellas en lo escrito, las autoras desarrollaron una clasificación que, como veremos a continuación, parte del nivel de mayor evolución al de menor logro:

a) Todo está escrito, incluso los artículos. Los niños hacen corresponder cada una de las partes del enunciado oral con las palabras del texto. Ellos infieren el lugar de las palabras mencionadas a partir de estrategias como la repetición para sí mismos del enunciado o el recuento de las porciones gráficas. Cabe observar que, aun cuando algunos niños son capaces de descifrar, esto es un recurso que aparece únicamente en momentos problemáticos, pero no como una verdadera alternativa a seguir.

b) Todo está escrito, excepto los términos funcionales. Los niños no reconocen estas formas ni en lo oral ni en lo escrito; por un lado, porque no las consideran palabras y, por otro, porque rechazan que las secuencias menores de tres letras puedan decir algo (Ferreiro \& Teberosky, 1979: 153-154). Por ejemplo, ante el enunciado Papá patea la pelota, un niño de 6 años ubica las palabras papá, patea y pelota en las posiciones respectivas, pero 
no reconoce la como palabra. Él afirma que es parte de pelota (Ferreiro \& Teberosky, 1979: 151).

c) Los sustantivos están escritos de manera independiente, pero el verbo es solidario de la oración entera, o del predicado entero. La dificultad central de este nivel, afirman Ferreiro y Teberosky (1979: 168), consiste en separar el verbo, debido a que al niño le resulta muy difícil de concebir la acción aislada del sujeto que la realiza o del objeto que la recibe. Así, con el mismo enunciado Papá patea la pelota, otro niño de seis años identifica pelota y papá en los lugares respectivos y escoge la para patea; luego corrige y dice: "acá patea", señalando la palabra correspondiente, y añade: "patea la pelota" (Ferreiro \& Teberosky, 1979: 157).

d) Imposibilidad de efectuar una separación entre las partes del enunciado que pueden hacerse corresponder con las partes del texto. Los niños no pueden concebir que en el texto esté escrita una parte aislada del enunciado (Ferreiro \& Teberosky, 1979: 170), como el caso de un niño de cinco años, a quien le cuesta admitir que en el enunciado escrito El oso come miel pueda decir oso, miel o come miel en alguna parte; sin embargo, cuando se le vuelve a preguntar qué dice, él afirma: "el oso come miel” señalando de corrido el enunciado.

e) Toda la oración está en un fragmento del texto; en el resto del texto, otras oraciones congruentes con la primera. Por ejemplo, una niña de cuatro años propone, para cada uno de los fragmentos del enunciado Papá patea la pelota (de izquierda a derecha): "papá patea la pelota", "papá grave", "papá escribe la fecha” y "papá se va a dormir". Según Ferreiro y Teberosky (1979: 172), las respuestas de tipo $d$ y $e$ se asemejan, porque ambas expresan la dificultad del niño para distinguir entre la totalidad del enunciado y sus partes. Se distinguen, sin embargo, porque los niños de esta categoría ubican el enunciado entero en uno o dos segmentos gráficos; en el resto interpretan otras oraciones, por lo general, relacionadas temáticamente con el enunciado modelo.

f) Ubicación exclusiva de los nombres en los fragmentos del texto; en el resto, eventualmente, otros nombres compatibles con los anteriores. Los niños pueden repetir los mismos sustantivos del enunciado oral o proporcionar nuevos para atribuir significado a cada parte de texto, como lo muestra el 
caso de una niña de cinco años con el enunciado Papá patea la pelota. Ella dice que "papá" está en patea la, "porque es más largo"; "pelota" en pelota, y en papá interpreta mamá, porque dice que hace falta (Ferreiro \& Teberosky, 1979: 174).

El conocimiento sobre los textos que los niños oyentes muestran tener antes de alcanzar una lectura convencional suscita un conjunto de expectativas acerca de las ideas que los que carecen de audición pudieran desarrollar en torno de la lengua escrita. Las investigaciones de Báez (2010) y Báez, Bellini, Biglione, Dotto, Fagoaga, Migliarini y Safón (2013) sobre la interpretación de textos acompañados de imágenes, efectuadas con escolares sordos no oralizados, hablantes de la lengua de señas argentina (LSA), dan cuenta de una evolución similar a la planteada por Ferreiro y Teberosky (1979) para los niños oyentes. Los niños sordos de esos estudios avanzan desde la indiferenciación imagen-texto hacia un análisis en el que "las características gráficas del enunciado escrito se constituyen en indicadores lingüísticos para su interpretación" (Báez, 2010: 20). Según los resultados de Báez et al. (2013), algunos niños llegan a considerar que todo lo enunciado está escrito (incluso los artículos) e identifican cada segmento de lo escrito.

Que los niños sordos construyan hipótesis de representación prealfabéticas de la misma manera que los oyentes abre otras rutas de investigación que permiten acercarnos al entendimiento de cómo aprenden los sordos las escrituras alfabéticas. En este estudio se examina el comportamiento de los niños sordos ante la lectura de enunciados que carecen de imágenes. La finalidad de probar la interpretación de textos sin imágenes es, como lo plantean Ferreiro y Teberosky (1979: 142), evaluar 'la posibilidad de 'deducir', en función de la información disponible (texto escrito, lectura del adulto y conocimiento lingüístico del niño), qué es lo que 'debe' estar escrito en cada fragmento". La situación con los niños sordos es interesante porque la lengua del texto (español escrito) y la lengua de la que se parte para interpretar los enunciados (LSM) son distintas. Se intenta, entonces, comparar, con respecto al esquema propuesto por Ferreiro y Teberosky (1979) para el aprendizaje de la lengua escrita de niños oyentes, qué tanto comparten o cuánto difieren las interpretaciones de los niños sordos y las de los oyentes que están aprendiendo a leer. 


\section{Metodología}

La investigación se realizó con 22 niños sordos profundos de una escuela primaria privada de la Ciudad de México, especializada en la enseñanza de la LSM; 15 de ellos son hombres y 7 son mujeres con un rango de edad entre 6 y 9 años; 7 cursaban cuarto grado; 7 , tercero; 5 , segundo y 3 , primero. Todos los niños recibían enseñanza de la lectura apoyada en LSM y habían aprendido a signar las letras.

Participaron únicamente sujetos competentes de LSM. Se seleccionaron con la ayuda de sus maestras y mediante una entrevista en la que se valoró su vocabulario y la comprensión de instrucciones relacionadas con el protocolo de entrevista. Según los datos asentados en los expedientes escolares, 15 tenían como lengua nativa la LSM, 6 habían aprendido simultáneamente LSM y señas caseras desde temprano; únicamente un niño había recibido enseñanza de LSM a partir de su entrada a la escuela. En cuanto a los padres, solo los de dos niños eran hablantes nativos de LSM; los padres de diez más habían empezado a aprenderla desde que sus hijos fueron detectados con sordera; los de seis de ellos decían saber un poco, y los restantes, nada (empleaban señas caseras).

La prueba de interpretación de textos sin imágenes se basó en el diseño de Ferreiro y Teberosky (1979), aunque con algunas variaciones. En este estudio, la situación de entrevista consistió en presentar a cada niño tres tarjetas con enunciados escritos en letra script. Estos se leían en LSM, uno a la vez (los textos y su interpretación en LSM se indican más adelante); concluida la lectura, se llevaba a cabo el interrogatorio, tratando de que la enunciación en LSM fuera lo más cercana posible al sentido de las preguntas en español. Las entrevistas fueron realizadas por un hablante e intérprete de LSM, dirigido por uno de los investigadores que comprendía la LSM. Estuvo también presente otro colaborador hablante e investigador de la LSM, quien supervisó la entrevista y su transcripción.

Fijar las preguntas para el interrogatorio en LSM fue ciertamente complicado, no solamente por las diferencias entre la marcas temporales, aspectuales y modales de los verbos en las dos lenguas, sino también porque las señas para expresar los verbos usados en las entrevistas con los oyentes produjeron respuestas inesperadas en los niños sordos. Decir, una seña cuyo movimiento parte de la boca, no siempre era interpretada por los niños como referida al texto; leer 
entrañaba la actividad de deletrear. Después de varias pruebas se optó por preguntar del modo siguiente:

¿Dónde está escrito X? ¿ ¿Está escrito X? ¿Dónde?

LSM: escribir $\mathrm{X}_{i \text { dónde? }}$

¿Qué está escrito acá? o ¿Qué dice acá?

LSM: (señalando sobre la tarjeta), acá qué significar (en realidad: qué seña tener).

Se eligieron enunciados que guardaran semejanza con los utilizados por Ferreiro y Teberosky (1979) y Ferreiro y Gómez Palacio (1982a). Además se tomó en consideración que fueran enunciados donde hubiera: 1) términos con equivalencias en lengua de señas; por ejemplo: los sustantivos pastel y casa, el adjetivo azul, el adverbio lejos, los determinantes posesivo y numeral (en realidad, el artículo indeterminado del español); 2) términos exclusivos del español: preposiciones, artículos y verbo copulativo, y 3) términos con particularidades en LSM, como el verbo comer, que para este enunciado integra la forma de lo comido, y el intensificador muy que se articula simultáneamente a lejos. Se utilizaron únicamente tres enunciados para no alargar demasiado la entrevista, ya que esta comprendía otras tareas del protocolo de evaluación del conocimiento de la lengua escrita. El orden de presentación de los enunciados durante la entrevista fue el que se muestra a continuación.

1) María come una rebanada de pastel.

LSM: María pastel uno comer + Clasificador: forma rectangular larga integrada en la seña para comer

2) La casa es azul.

LSM: casa azul

3) Su casa está muy lejos.

LSM: su casa lejos + Intensificador: labios protruidos en simultáneo al adverbio 
Además de las diferencias relativas a los términos que integran los enunciados en ambas lenguas, hubo otras que valía la pena investigar. El primer enunciado en LSM sigue el orden sov, el cual resulta ajeno al español. ${ }^{1}$ Por otra parte, este mismo enunciado contiene un verbo y un sustantivo que requieren clasificadores. En LSM, los clasificadores se añaden a los sustantivos para indicar la forma (redondo, cuadrado, etc.) y la dimensión de los objetos denotados (tamaño, longitud, espesor, etc.); así también hay clasificadores que pueden agregarse al verbo para precisar propiedades del objeto del que se predica (Cruz Aldrete, 2009). La configuración manual (CM) del clasificador se realiza en el momento de la predicación, combinada con el morfema raíz de movimiento (Orduña \& Ruiz, 2010). Así, en el enunciado maría pastel uno comer el verbo comer conserva el movimiento original, pero la $\mathrm{CM}$ se modifica para indicar que el objeto que se come es rectangular y alargado (la rebanada).

En español, la predicación nominal y la adjetiva requieren de un verbo copulativo. En cambio, en LSM la relación entre sujeto y atributo se expresa por medio de la yuxtaposición de dos frases nominales o una frase nominal y una adjetiva (Cruz Aldrete, 2009). Así, el enunciado La casa es azul se constituye por dos señas, una para casa y otra para azul. En el enunciado Su casa está muy

1 El orden de los constituyentes de la oración ha sido muy discutido tanto en la ASL como en la LSM y otras más. Liddell (1980) sostiene que la estructura básica de la LSA es Svo, aunque admite también excepciones a la misma. Autores como Janzen (1999) se inclinan más por una perspectiva pragmática que prioriza la estructura tópico-comentario como rectora del orden en la LSA. De manera general, el orden de la LSM se ha descrito como sov (Quinto, 1999, citado en Cruz Aldrete, 2009); sin embargo, hay autores que documentan el orden svo (Fridman, 2005) y otros que argumentan en favor de la estructura tópico-comentario (Sutton Spence \& Woll, 1999, citado en Cruz Aldrete, 2009). Ahora bien, aunque el orden svo es el que se observa en la mayoría de los enunciados, aparecen también las estructuras sov, osv, vos, vso y ovs según el tipo de verbo que se utilice (Cruz Aldrete, 2009). De acuerdo con esta última propuesta, la clase de verbos (llanos y demostrativos) puede influir en el orden de constituyentes que presenta la LSM. El orden de los constituyentes varía según la lengua de que se trate. Bobillo García, Morales López, Reigosa Varela, Freire Rodríguez y Pérez Casanova (2004) destacan que para la lengua de señas española (LSE) (variante de La Coruña) el orden no marcado es Sov y la estructura de tópico gramaticalizado, en contraste con el orden svo que aparece solo en ciertos contextos. Citan además el orden no marcado de otras variedades de LS utilizadas en países hispanohablantes: sov para la LSA, svo para la lengua de señas colombiana (LSC) y SVO para la lengua gestual portuguesa (LGP). 
lejos hay otras diferencias: en su realización hay un rasgo no manual (labios protruidos) que se ejecuta simultáneamente a la cM lejos y equivaldría al adverbio del español muy.

\section{Resultados}

El análisis de las respuestas de los niños sordos arroja datos que no coinciden del todo con el esquema propuesto por Ferreiro y Teberosky para el desarrollo de la lectura de los niños oyentes. Si bien las respuestas de los niños sordos pueden ubicarse en categorías semejantes a las $a, b$ y $f$ de Ferreiro y Teberosky, aunque con diferencias que se comentarán enseguida, no aparecen respuestas propias de las categorías $c, d$ y $e$. Lo que sí se observa es una evolución de las respuestas en la que las partes de lo escrito (las palabras gráficas del sujeto alfabetizado) cobran cada vez mayor importancia.

\subsection{Categorización de las respuestas y análisis de casos}

Las categorías se construyeron a partir del examen de las respuestas de los sujetos. Los parámetros de clasificación tuvieron en cuenta tanto los elementos del texto identificados como el lugar donde los niños ubicaron el verbo comer (en realidad, el único verbo pleno que figura en los enunciados proporcionados). En su presentación se ha decidido preservar el orden descendente que siguieron Ferreiro y Teberosky para facilitar las comparaciones.

a) Intentan interpretar todas las partes del enunciado escrito siguiendo el orden sintáctico del español.

b) Intentan interpretar todas las partes del enunciado escrito siguiendo el orden de la LSM.

c) Interpretan los términos léxicos y algunos determinantes que pueden hacer corresponder con señas siguiendo el orden sintáctico del español.

d) Interpretan los términos léxicos y algunos determinantes que pueden hacer corresponder con señas siguiendo el orden de la LSM.

e) Interpretan solamente términos léxicos en orden diverso. 
3.1.1. Casos del tipo a) intentan interpretar todas las partes del enunciado escrito siguiendo el orden sintáctico del español

Los sujetos agrupados en esta categoría tratan de atribuir un significado a cada parte escrita, a pesar de que los componentes de los enunciados en la LSM son menos que en español. Su interpretación de los términos funcionales no siempre se corresponde con el significado que tienen en español; por ejemplo, ellos tratan las preposiciones con una seña genérica enseñada en la escuela y que las maestras denominan cual, y los artículos, con la seña del demostrativo, que es la forma con que los identifican las maestras. La interpretación del orden de los argumentos siempre la realizan de acuerdo con el orden del enunciado en español (svo). Una característica más de los sujetos que componen este grupo es que anticipan, sin que se les requiera, la lectura de uno o más enunciados. El primer enunciado fue anticipado correctamente por tres niños y uno más que lo hizo de manera aproximada, mientras que el segundo y el tercer enunciado fueron anticipados correctamente por cuatro niños. Son cinco los niños clasificados en este conjunto, cuatro de cuarto grado y uno de tercero. Para ilustrar su comportamiento, se describe lo que hicieron algunos de ellos con el primer enunciado.

Una niña de cuarto grado anticipa la lectura del enunciado María come una rebanada de pastel a la del entrevistador; lee mediante dactilología: $M-a-r-i-a$, en LSM comer uno, con dactilología $r-e-b-a-n-a-d-a$ y en LSM cual pastel. Después de recibir la lectura del investigador en LSM, ubica en el enunciado escrito los términos que tienen sentido en su lengua. Ella puede entender la separación de comer (seña sin clasificador) y rebanada (CM de rebanada dirigida hacia la boca) que realiza el investigador y relacionar ambas señas con las partes del enunciado apropiadas.

Un tanto distinto es el caso de otro niño de cuarto grado, quien anticipa en LSM la lectura del primer enunciado como: María pastel rebanada-comer gran$d e$. Recibe la lectura del entrevistador y, al preguntársele en dónde están escritas las partes del enunciado proporcionado, localiza María, come y uno en las palabras correspondientes; ubica pastel en rebanada; interpreta de como cual, pero no puede asignar ningún significado a la palabra pastel. Para él rebanada es un término conflictivo que le resulta difícil separar de la seña comer. 
El caso de otra niña de cuarto grado difiere de los dos anteriores porque ella no anticipa la interpretación del primer enunciado. Tampoco parece tener conflicto alguno con las partes. Cuando se le proporciona la interpretación, asigna a cada palabra gráfica el significado correspondiente; incluso se percata de la preposición, que interpreta como cual, de acuerdo con lo que se la ha enseñado en la escuela.

El enunciado La casa es azul resultó ser menos complicado de interpretarse, pese a contar con dos elementos que no figuran en la lengua de señas. Todos los niños anticiparon su lectura y le asignaron una seña a cada palabra escrita. El artículo la fue interpretado con la seña para el demostrativo y el verbo copulativo con una seña creada especialmente para marcarlo. Solamente hubo un niño que no pudo reconocer el verbo ser; él identificó todos los elementos del primer enunciado, pero en el segundo leyó: ese casa azul. En el momento de preguntarle dónde está cada una de las partes es cuando se percata del copulativo y lo deletrea, pero no reconoce ningún significado para el término.

El enunciado Su casa está muy lejos también fue leído anticipadamente por varios niños. Durante el interrogatorio, todos ellos lograron interpretar sin dificultades el posesivo y situarlo al inicio del enunciado escrito; del mismo modo, encontraron casa y lejos pero algunos tuvieron problemas con la identificación de está, que leyeron dactilológicamente.

En suma, los niños de este grupo intentan interpretar todas las partes escritas, incluso las preposiciones y copulativos, aunque no se correspondan con ningún elemento en su lengua, y las ubican en los lugares correspondientes. Todos ellos le atribuyen a la preposición de la seña $\mathrm{cual}$, sea durante la interpretación anticipada o en el momento del interrogatorio; casi todos tienen una seña para los copulativos, y, quienes no la conocen, saben que es y está pueden leerse, porque deletrean dichos términos, y entran en conflicto al no poder asignarles un significado. Una última observación es que tres de los niños que anticipan la lectura leen dactilológicamente las palabras cuyo significado no reconocen en su lectura anticipada. Notoriamente, el deletreo manual les permite identificar las letras que las componen, pero no su significado. 


\subsubsection{Casos del tipo b) intentan interpretar todas las partes del enunciado escrito siguiendo el orden de la LSM}

El comportamiento de los niños de esta categoría es muy parecido al de la anterior. Ellos pueden anticipar la lectura del segundo y tercer enunciado de manera más o menos exitosa y hacer corresponder las partes que enuncian con lo que está escrito. Así, identifican la posición de los sustantivos, adjetivos, adverbios, determinantes e, incluso, los copulativos en el enunciado escrito. Sin embargo, se comportan de manera diferente en relación con el primer enunciado, María come una rebanada de pastel. Aunque también intentan dar una interpretación a todas las partes gráficas que lo componen, colocan el verbo al final del enunciado y el término pastel lo ubican en el lugar de la palabra comer, apegándose de este modo al orden que tiene el verbo en la LSM. Hay en este grupo dos niños de cuarto grado y dos de tercero.

El caso más notable es el de un niño de tercer grado, pues es el único que identifica en la lectura todos los términos del enunciado escrito María come..., incluida la versión de la preposición que se enseña en la escuela (él lee: María comer uno rebanada cual pastel). Sin embargo, durante el interrogatorio coloca el verbo al final. Para este niño, el orden sov parece tener más fuerza que el del español, presente en el texto.

El comportamiento de una niña, también de tercero, es semejante al que se acaba de presentar. Aunque en su lectura del segundo y tercer enunciado puede relacionar los términos funcionales escritos con elementos de la LSM, o con las señas especiales cual y ser, cuando se trata de ubicar el lugar del verbo en el enunciado María come una rebanada de pastel, lo pone al final. Es solo después de que el entrevistador insiste en preguntar por la ubicación de comer y pastel que corrige y localiza su lugar en el texto.

3.1.3. Casos del tipo c) interpretan los términos léxicos y algunos determinantes que pueden hacer corresponder con señas siguiendo el orden sintáctico del español

Dentro de esta categoría están los niños que todavía no pueden interpretar ni atribuir señas que no son parte de su lengua materna a las partes correspondientes 
del enunciado escrito. Los niños encuentran en el texto las partes que se corresponden con los sustantivos, adjetivos y verbos plenos; así también, pueden hacer corresponder los determinantes del español con los demostrativos de la LSM, pero no las marcas copulativas ni la preposición. Ellos parecen ceñir su interpretación a lo que puede hacerse corresponder con lo que tienen en la lengua de señas.

Otra característica importante es que pocos de ellos intentan anticipar alguna interpretación de los enunciados. Cuando lo hacen, es siempre del segundo o tercer enunciado y nunca completa. Son cinco los niños agrupados en esta categoría: tres de tercer grado y dos de segundo. Enseguida se describen algunos casos para ilustrarla.

Una niña de tercer grado deletrea manualmente pero no puede interpretar el primer enunciado. Después de la lectura del entrevistador, asigna a cada parte del enunciado escrito el significado correspondiente, a excepción de la preposición, que no reconoce. Ella pone atención a las propiedades gráficas del texto, pues comenta las semejanzas de lo escrito con otras palabras; por ejemplo, dice que $r-e-b-a-n-a-d-a$ (con deletreo manual) se parece a $n-a-d-a$ y $n-a-d-a-r$ (también con deletreo manual) y que a come le falta la $r$. El segundo y tercer enunciado le resultan más conflictivos. Ella los intenta leer pero no puede completarlos. En $L a$ casa es azul reconoce el determinante, el sustantivo y el adjetivo pero no la forma es ni su seña ser. En Su casa está muy lejos anticipa su casa y solo después de recibir la interpretación del entrevistador reconoce muy y lejos pero no está, que puede deletrear, pero que, como carece de significado en su lengua, no puede admitir que diga algo.

Los restantes niños asignan María, come, una y pastel a partes del enunciado. De ellos, solamente una niña, también de tercer grado, puede encontrar la ubicación correcta de cada término, incluso rebanada. Otro niño de tercero ubica inicialmente pastel en la palabra escrita come, luego duda y la localiza en rebanada; reubica come en comer; entonces cambia de lugar pastel y lo asigna en el lugar correcto, pero no sabe qué hacer con rebanada. Por último, los dos niños de segundo asignan pastel a rebanada y, después de preguntárseles por rebanada, la ubican en pastel.

Es en la interpretación de los enunciados que contienen las marcas copulativas donde estos niños tienen los mayores problemas. En el enunciado La casa es azul, únicamente el niño de tercer grado, quien había anticipado la lec- 
tura casa azul, asigna la interpretación ese a la y también al verbo ser. Los restantes niños de tercero localizan casa, azul y asignan ese a la, pero no reconocen el copulativo.

En el enunciado Su casa está muy lejos, todos reconocen el posesivo, excepto una niña de segundo. Ella asigna el posesivo a la palabra casa, después ubica casa en la posición correcta y, cuando se le pregunta de nuevo por el posesivo, vuelve a colocarlo en casa; sin embargo, interpreta correctamente el adverbio muy y el adjetivo lejos. Lo contrario sucede con la otra niña de segundo grado, quien señala correctamente el sustantivo casa y el posesivo, pero no logra interpretar muy ni lejos. Ella interpreta en un inicio lejos como lugar y deletrea $e-s-t-a ́$, pero cuando recibe la interpretación en lengua de señas, admite no reconocer las señas para los dos últimos términos y se niega a ubicarlos en el enunciado escrito.

Es posible observar cómo los niños de este grupo están empezando a considerar algunos términos funcionales. Todos interpretan el artículo indefinido una en el primer enunciado, posiblemente debido a su similitud con el numeral en LSM; los de tercero interpretan la como ese en el segundo enunciado, y algunos más el posesivo y los adverbios. Las dificultades presentes en la interpretación de los enunciados con copulativos, el desconocimiento de la preposición de en el primer enunciado y el que los niños no puedan anticipar una lectura completa de ningún enunciado muestran el comportamiento diferente de este grupo en relación con la categoría $a$.

\subsubsection{Casos del tipo d) interpretan los términos léxicos y algunos determinantes que pueden hacer corresponder con señas siguiendo el orden de la LSM}

Los niños que integran esta categoría pueden deducir los términos léxicos y algunos de ellos aislar uno que otro determinante. En el primer enunciado separan el verbo, así como los sustantivos núcleos del sujeto (María) y el objeto (pastel). Sin embargo, colocan los términos que identifican de acuerdo con el orden que tienen en la lengua de señas. Ellos ubican sistemáticamente el nombre María al inicio del enunciado y asignan el verbo comer a la última palabra del texto. Son cuatro niños los que forman parte de este grupo: uno de cuarto grado, uno de tercero y dos de segundo. A continuación se detallan algunos comportamientos. 
El desempeño del niño de cuarto no es igual al de sus compañeros de grado, que pueden reconocer todos o casi todos los términos funcionales, sea de acuerdo con el orden del español o el de la LSM. El que no intente anticipar la interpretación del primer enunciado, no reconozca la preposición y sitúe el verbo al final del mismo lo colocan en este grupo. Asimismo, su lectura de los restantes enunciados, que se apega más a la cantidad de elementos de la lengua de señas que a la del texto, es otro argumento para considerarlo dentro de este grupo. No obstante, este niño puede reconocer algunos términos funcionales; así, cuando se le pide identificar el artículo la, en el segundo enunciado, agrega un elemento más en su interpretación, el determinante ese.

El niño de tercer grado tiene más dificultades para identificar las partes del enunciado. En el primer enunciado, ubica María, una y rebanada siguiendo el orden de las palabras escritas, pero a pastel y come les da el orden de la LSM. En el segundo enunciado, reconoce los lugares de las palabras casa y azul para los mismos términos, aunque lo hace en lugares invertidos. En el tercero, localiza su, casa, muy y lejos, pero en desorden; incluso, selecciona está dos veces para ubicar primero el posesivo y después el adverbio lejos. Sin embargo, no selecciona la palabra escrita $s u$, posiblemente porque tiene solo dos letras y estén operando restricciones de cantidad.

El niño y la niña de segundo grado reconocen aún menos términos en los enunciados escritos. En el primer enunciado, el niño admite María, pastel y come, en tanto que la niña, María, rebanada y comer. Pareciera que ambos niños solamente consideran los sustantivos que identifican quien come y el objeto que se come. El verbo, que también puede estar escrito, lo ponen al final del texto. En el segundo enunciado ambos reconocen casa y azul. Pero el enunciado Su casa está muy lejos les resulta más complicado. El niño admite más términos que la niña: identifica su, casa y lejos en las palabras escritas su, casa y está. Al preguntársele por los sobrantes gráficos, les atribuye significados que pueden relacionarse con los de lejos (el niño atribuye cansando a la palabra muy y casa, tranquila a lejos). La niña únicamente toma el término lejos y lo ubica donde está casa.

Cabe observar que el comportamiento de los sujetos que se agrupan en esta categoría no es tan uniforme como el de los que integran las restantes. Por un lado, los dos niños de los grados superiores logran identificar más elementos 
que los dos de segundo grado, especialmente en el segundo y tercer enunciado, aunque no tantos ni de manera tan sistemática como los de la categoría $b$. Por otro, la interpretación de los dos niños de segundo grado en relación con tales enunciados está más cerca de lo que hacen los niños de la categoría $e$. Sin embargo, es ante el primer enunciado escrito: colocar el sujeto en la primera posición y el verbo en la última, que su comportamiento se iguala. Este parámetro distingue a los dos niños de segundo grado de los de la categoría que se verá a continuación.

\subsubsection{Casos del tipo e) interpretan solamente términos léxicos en orden diverso}

Los niños que integran este grupo identifican los términos léxicos pero no los determinantes, preposiciones ni adverbios. Parecen estar todavía lejos de hacerlo, pues no aciertan a deducir todos los términos léxicos ni a darles un orden consistente cuando tratan de hacerlos corresponder con las palabras escritas. Esto muestra una diferencia importante con respecto del grupo anterior, porque los niños de esa categoría asignan sistemáticamente el sustantivo sujeto a la primera posición del enunciado y el verbo a la última, al menos con el primer enunciado. Hay cuatro niños en este grupo, uno de segundo y tres de primer grado. Enseguida se describe lo que hacen algunos de ellos durante las entrevistas.

El niño de segundo grado reconoce el verbo, los sustantivos del sujeto y objeto, y el numeral, además de su lugar en el enunciado escrito. En el segundo enunciado solamente logra ubicar casa. Ante las preguntas del entrevistador, él responde que no sabe qué más pueda estar escrito en las restantes partes del enunciado.

De manera semejante, uno de los niños de primero admite que María, pastel y comer están escritos, pero los ubica en las palabras rebanada, pastel y una, respectivamente. En el segundo enunciado acepta casa y azul y las ubica correctamente, pero cuando se le pregunta por las partes restantes del enunciado vuelve a colocarlas en la y es. Este comportamiento es una variante del que tiene el niño de la categoría anterior que asigna otros significados a las partes gráficas, pues tampoco sabe qué hacer con los sobrantes.

Los otros dos niños admiten menos elementos todavía. En el primer enunciado, uno solo reconoce que están escritos comer, pastel y rebanada y las lo- 
caliza del siguiente modo: pastel, primero en María y después en pastel; comer en una, y rebanada otra vez en María. El otro solo acepta los sustantivos María y pastel, que ubica en las dos primeras palabras del enunciado escrito; después se niega a seguir identificando más términos. En el segundo enunciado, ambos reconocen un solo término, casa, y lo ubican en lugares equivocados: uno en la y el otro en más de un lugar, pero desiste ante la presión del entrevistador. Finalmente, este último niño decide ubicar azul en es, después de que el investigador reitera la pregunta de si puede estar escrito dicho término. Es importante señalar que la entrevista resultó bastante difícil en este grupo, por lo que se decidió trabajar solamente con dos enunciados para cada uno de los niños.

\subsection{Resultados globales}

El orden propuesto para las categorías de respuesta representa el progreso en la interpretación de los enunciados escritos que logran los niños sordos entrevistados. La categoría a constituye la fase más avanzada de interpretación de la lengua escrita, puesto que los niños ubicados en ella son los que más elementos de lo escrito interpretan y, aunque no comprendan el significado de todos ellos en español, admiten que puedan tener un orden diferente al de la LSM. En contraste, la categoría $b$ no denota igual grado de progreso, pues si bien los niños que la integran también intentan identificar todas las partes gráficas, adjudican al verbo un orden que no se corresponde con el de la lengua escrita sino con el de la LSM. Las restantes categorías involucran un menor logro interpretativo en relación con las partes del enunciado escrito: $c$ y $d$ son equivalentes en términos de la cantidad y calidad de los elementos que los niños admiten, pero en $d$ vuelve a observarse la influencia del orden Svo de la LSM. La categoría $e$ es donde están los niños que solo reconocen los sustantivos como parte de lo escrito y los ubican en orden diverso.

Con la finalidad de examinar si existe correlación entre la progresión de las categorías de respuesta y el grado escolar cursado, en el Cuadro 1 se presenta la distribución de los niños en tales categorías. Se muestra la tendencia a alcanzar categorías más evolucionadas a medida que se avanza en los grados escolares. Así, en la categoría $a$ se encuentran niños de los dos grados superiores (uno de tercero y cuatro de cuarto) y lo mismo sucede en la categoría $b$, que involucra 
un similar tipo de análisis del enunciado escrito. En las categorías $c$ y $d$, equivalentes en cuanto al análisis del enunciado, aparecen dos niños de segundo grado, uno de tercero y uno de cuarto. ${ }^{2}$ Por último, en la categoría $e$ solo aparecen niños de primer y segundo grado. Así, pues, salvo por el niño de cuarto grado que aparece en la categoría $d$, se constata una distribución de los sujetos escalonada. Notoriamente, en las categorías extremas se observa que los niños comprendidos en ellas pertenecen a grados excluyentes.

Cuadro 1. Distribución de los niños por grado escolar en las cinco categorías de respuesta

\begin{tabular}{|c|c|c|c|c|}
\hline Categoría & $1^{\circ}$ & $2^{\circ}$ & $3^{\circ}$ & $4^{\circ}$ \\
\hline $\begin{array}{l}\text { a) Interpretan todo lo escrito siguiendo el } \\
\text { orden sintáctico del español }\end{array}$ & & & 1 & 4 \\
\hline $\begin{array}{l}\text { b) Interpretan todo lo escrito siguiendo el } \\
\text { orden de la LSM }\end{array}$ & & & 2 & 2 \\
\hline $\begin{array}{l}\text { c) Interpretan léxico y algunos elementos } \\
\text { funcionales siguiendo el orden sintáctico } \\
\text { del español }\end{array}$ & & 2 & 3 & \\
\hline $\begin{array}{l}\text { d) Interpretan léxico y algunos elementos } \\
\text { funcionales siguiendo el orden de la LSM }\end{array}$ & & 2 & 1 & 1 \\
\hline $\begin{array}{l}\text { e) Interpretan solo términos en léxicos en } \\
\text { orden diverso }\end{array}$ & 3 & 1 & & \\
\hline
\end{tabular}

\section{CONCLUSIONES}

Los resultados muestran cómo los niños sordos atribuyen significado a las partes que componen un enunciado escrito, sea que ellos puedan anticipar su lectura, sea que tengan que recibir la interpretación de un lector. De manera similar a los oyentes, en un principio piensan que la escritura remite a la lengua, aunque solo

2 Cabe señalar, por un lado, que las dificultades de este niño de cuarto grado se extienden a todas las situaciones en que participó y, por otro, que su desempeño en lengua de señas no era igual al de los niños de su edad, posiblemente porque comenzó a aprender la lengua de señas al ingresar al sistema escolar.

Estudios de Lingüística Aplicada, año 35, número 66, diciembre de 2017, pp. 77-107 doi: 10.22201/enallt.01852647p.2017.66.829 
a los elementos con significación plena, como los sustantivos y algunos adjetivos o verbos. Así también, en la medida en que interactúan con la lengua escrita, tienen en cuenta cada vez más detalladamente las propiedades del texto, tanto cuantitativas (cantidad de las secuencias gráficas) como cualitativas (el orden de las secuencias y las letras que las componen). Sin embargo, pese a que muchas de sus respuestas comparten rasgos con las de los oyentes, hay otras que resultan distintas. Por ello, las categorías en que se han agrupado no se corresponden del todo con las definidas por Ferreiro y Teberosky (1979).

La categoría que en este estudio denota un mayor conocimiento de la lengua escrita presenta diferencias relevantes respecto de las investigaciones precedentes (Ferreiro \& Teberosky, 1979; Báez et al., 2013). En estos trabajos se establece que los niños que pertenecen a esa categoría admiten que todo lo que se enuncia cuando se lee está escrito en el texto. Desde la perspectiva del hablante sordo esto es difícil, porque no se puede hacer corresponder lo que se expresa en lengua de señas con todo lo escrito. Las estructuras de la lengua del texto, español, y de la lengua mediadora de la interpretación, LSM, son muy distintas. Sin el conocimiento lingüístico suficiente sobre la lengua de la escritura no es factible que el niño sordo pueda identificar cada parte del enunciado escrito.

Lo que sí vemos es cómo los niños sordos atribuyen los significados de su lengua a las partes escritas y, en la medida en que incrementan su interacción con la escritura, prestan mayor atención a las propiedades del texto e intentan interpretar todas sus partes. Por ejemplo, ellos encuentran la palabra para el clasificador (rebanada) y asignan valor de demostrativo a los artículos. También pueden reconocer las preposiciones o el verbo copulativo; sin embargo, es posible que no les den el significado que tienen en español. Al parecer su interpretación depende de señas con valor fijo, provistas por la escuela a la que asisten, y sobre las cuales es necesario investigar para entender cómo contribuyen o interfieren en la interpretación global de los enunciados, precisamente porque no reciben enseñanza especializada del español como segunda lengua.

Hay otros dos grupos, correspondientes a las categorías $b$ y $d$ que también distan de lo que presentan los estudios citados. Son los niños que sistemáticamente ubican el verbo al final del enunciado María come una rebanada de pastel, independientemente de que traten de interpretar todas las partes escritas o solo lo que reconocen en la lengua de señas. La posición del verbo al final de la 
oración presentada es una característica de la lengua de señas que interviene de manera tal en la interpretación de ese enunciado que aun los niños que tienen en cuenta las propiedades cuantitativas y cualitativas del texto para anticipar una interpretación adecuada asignan el verbo a la palabra final.

Quedan, por último, los niños que solo pueden aceptar los términos léxicos - categoría e y los dos niños de segundo grado de la categoría $d$. Ellos identifican sustantivos, adjetivos y, salvo uno, todos reconocen el verbo comer, aunque no los ubiquen en los lugares correspondientes. En este estudio no aparecen niños que no pudieran separar el verbo de los sustantivos sujeto u objeto. Tampoco hay niños que no pudieran siquiera diferenciar las partes del enunciado de la totalidad. Lo que sí se encuentra son niños que no pueden separar el clasificador (rebanada) del verbo comer: tres de la categoría $e$, dos de la $d$ y uno de la $c$. Valdría la pena investigar si el clasificador tiene algún papel en la segmentación del verbo, así como las razones que llevan a los niños a admitir que clasificador y verbo pueden separarse, cuando en la LSM constituyen una unidad.

Los datos de este estudio no permiten establecer una progresión clara entre las categorías. Si bien entre las categorías $a, c$ y $e$ puede observarse gradación, en la medida en que hay mayor reconocimiento de las partes escritas, están las categorías $b$ y $d$, que también son previas a la $a$, en el sentido de que los sujetos que las integran no logran la identificación de todas palabras escritas. Es posible, entonces, que el desarrollo del conocimiento de la lengua escrita en los niños sordos tome más de una vía, debido a la fuerza que adquiere la lengua de señas en tanto mediadora de lo escrito. De este modo, los niños podrían pasar de la categoría $e$, en donde el lugar para ubicar las partes es inestable, a la $d$ (orden LSM), y de esta a la $c$ (orden español) o a la $b$ (orden LSM), antes de llegar a la $a$. Este es otro punto que habría que investigar, probando más enunciados Sov y otros órdenes característicos de la LSM.

En cuanto al tiempo de la progresión, se observan también diferencias. En los estudios de Ferreiro y Teberosky (1979) y Ferreiro y Gómez Palacio (1982a) el avance de los niños a través de las categorías de respuesta se logra por lo general en el transcurso de un año escolar. En este estudio no se pudo verificar ese lapso, ya que no hubo seguimiento longitudinal. No obstante, los datos transversales muestran cómo a los niños sordos les toma más tiempo aceptar que cada parte gráfica tiene un significado. La distancia entre los niños que intentan asig- 
nar un significado a cada palabra gráfica (niños de tercero y cuarto de primaria) y los que solo atribuyen términos léxicos a algunas partes escritas (niños de primero y segundo) es en promedio de dos grados escolares.

Dos reflexiones se desprenden de los resultados obtenidos. La primera refiere a la manera como los niños sordos se relacionan con la lengua de la representación escrita. Si bien para ellos la escritura refiere a los signos de la lengua, no es con los del español que se establece en un inicio. Los niños relacionan inicialmente la lengua escrita con la LSM, hecho que ha sido señalado por Goldin Meadow y Mayberry (2001), Allman (2002) y Morford, Wilkinson, Villwock, Piñar y Kroll (2011), entre otros investigadores. No obstante, a medida que avanzan en la escolaridad, comienzan a admitir formas que no son parte de la LSM e intentan interpretarlas, sea utilizando signos de la LSM en las posiciones posibles, sea aprendiendo de memoria señas especiales para los términos del español. Si la enseñanza sistemática del español escrito como segunda lengua se iniciara durante este periodo temprano, los niños sordos tendrían más posibilidades de alcanzar una interpretación literal adecuada; podrían comenzar a aprender la morfología y sintaxis que caracterizan la lengua que ven, en lugar de asociarlos con formas o señas que poco ayudan a comprenderla y, consecuentemente, a distinguirla de la lengua de señas.

La segunda reflexión tiene que ver con cómo los sordos se aproximan a la comprensión del sistema de representación, la escritura. Como se ha mostrado, mucho antes de lograr la lectura convencional, los niños sordos ya toman en consideración las propiedades gráficas de los textos. El comportamiento de los niños de las categorías más avanzadas pone de manifiesto cómo las propiedades cuantitativas de los textos comienzan a impactar su concepción de lo escrito. Ellos intentan dar un significado a partes gráficas que no tienen correspondencia con la interpretación inicial del enunciado en LSM. Las propiedades cualitativas también cobran importancia a medida que los niños avanzan en escolaridad. La lectura anticipada, parcial o completa, de los niños de las categorías $a$ y $b$, así como el que puedan asignar los términos interpretados a las palabras gráficas correspondientes, lo denota.

La manera como las propiedades cualitativas entran en juego en el aprendizaje de los sordos de la lengua escrita pone de relieve lo que les resulta pertinente durante el desarrollo del proceso. Si bien en los niños oyentes, centrarse en 
las propiedades cualitativas del texto involucra conocer las letras y descifrarlas, o bien, tomarlas como indicio para identificar las palabras del enunciado escrito (Ferreiro \& Gómez Palacio, 1982b), para los niños sordos, el reconocimiento de las letras no conduce al encuentro de la significación del texto. Por lo contrario, parece oponerse a ello: todos los niños de este estudio saben signar las letras y deletrean prácticamente sin errores cada una de las palabras que componen los textos, pero utilizan el deletreo únicamente cuando no reconocen las palabras. El deletreo manual no parece ser una actividad de la que los niños sordos puedan extraer ventajas, por lo menos no en los momentos iniciales de la alfabetización. Este fenómeno guarda semejanza con el deletreo de los niños oyentes que aparece cuando empiezan a recibir instrucción formal sobre la lectura. Ferreiro y Teberosky (1979: 132) muestran cómo esta conducta, producto de las prácticas escolares, centra la atención de los niños en la sonorización de las letras y los aleja de la interpretación del texto, creando un "divorcio entre descifrado y sentido".

Como sucede con los oyentes, es la interacción con la lengua escrita, es decir, la experiencia que proporciona la vista del texto y el conflicto que suscita su interpretación (tanto la que puede dar el niño, como la del mediador que sabe), lo que lleva a los sordos a aceptar que todo lo que se enuncia puede estar escrito, pero además les permite percatarse de las formas ajenas a su lengua. Para los sordos, la lengua escrita es una segunda lengua de naturaleza exclusivamente visual, que deben aprender a leer de una manera que les permita sortear la pronunciación. Su situación guarda muchas semejanzas con las de los aprendices del latín en la Edad Media; particularmente, los hablantes de lenguas celtas y germánicas, quienes durante siglos se alfabetizaron y aprendieron simultáneamente como segunda lengua sin recurrir a las correspondencias sonoras (Parkes, 1993). Son, precisamente, la condición de segunda lengua y el carácter puramente visual de la representación escrita lo que no se debe perder de vista en el desarrollo de futuras investigaciones sobre la adquisición de la lengua escrita en los sordos.

\section{REFERENCIAS}

Alegría, Jesús, \& Domínguez, Ana Belén (2009). Los alumnos sordos y la lengua escrita. Revista Latinoamericana de Educación Inclusiva, 3(1), 95-111. Recuperado de www.rinace.net/ rlei/numeros/vol3-num1/art7.pdf

Estudios de Lingüística Aplicada, año 35, número 66, diciembre de 2017, pp. 77-107 doi: 10.22201/enallt.01852647p.2017.66.829 
Alegría, Jesús, \& Lechat, Josiane (2005). Phonological processing in deaf children: When lipreading and cues are incongruent. Journal of Deaf Studies and Deaf Education, 10(2), 122133. Recuperado de https://www.researchgate.net/publication/7957255_Phonological_Processing_in_Deaf_Children_When_Lipreading_and_Cues_Are_Incongruent

Allman, Tamby M. (2002). Patterns of spelling in young deaf and hard of hearing students. American Annals of the Deaf, 147(1), 46-64. Recuperado de https://www.researchgate.net/publication/11313575

BÁEz, MóniCa (2010). Reflexiones acerca de la alfabetización de sujetos sordos. Avances de investigación psicolingüística. Lectura y Vida, 1, 18-27.

Báez, Mónica, \& Massone, María Ignacia (2012). La psicogénesis de la escritura en niños sordos: la interpretación de oraciones acompañadas con imagen. En María Ignacia Massone, Virginia Luisa Buscaglia \& Sandra Cvejanov (Comps.), Estudios multidisciplinarios sobre las comunidades sordas. Mendoza: Universidad Nacional de Cuyo.

Báez, Mónica; Bellini, Sandra; Biglione, Verónica; Dotto, Gabriela; Fagoaga, CaroliNa; Migliarini, Paula, \& SAfón, Omar (2013). La psicogénesis de la escritura en niños sordos no oralizados: la construcción de la noción de palabra. En S. Cvejanov (Dir.), Lengua de señas: estudios de lingüística teórica y aplicada (pp. 167-178). Neuquén: Universidad Nacional del Comahue. Recuperado de http://rdi.uncoma.edu.ar/bitstream/handle/123456789/1570/ Sandra\%20B.\%20CVEJANOV_lengua\%20de\%20se\%C3\%B1as.pdf?sequence=2\&isAllowed=y

Bobillo García, Nancy; Morales López, Esperanza; Reigosa Varela, César; Freire RoDríguez, Cristina, \& Pérez Casanova, Cristina (2004). El orden de los constituyentes de la oración declarativa en la LSE. En cultura-sorda.org. Recuperado de http://www.cultura-sorda.org/el-orden-de-los-constituyentes-de-la-oracion-declarativa-en-la-lse/\#_ftn1

Burgat, SANDrine (2009). Un cas spécifique de bilinguisme: l'enfant sourd locuteur de la langue des signes qui apprend à lire et à écrire en français. Rusca. Recuperado de http://www.msh-m. fr/article.php3?id_article $=655$

Cruz Aldrete, Miroslava (2009). Gramática de la lengua de señas mexicana. Estudios de Lingüística del Español, 28. Recuperado de http://elies.rediris.es/elies28/

FABBREtTi, DANiela (1997). Scrivere e segnare: la costruzione del discorso nell'italiano scritto e nella lingua italiana dei segni delle persone sorde (Tesis doctoral inédita). Universitá degli Studi di Roma, Roma.

Ferreiro, Emilia (1998). Alfabetización: teoría y práctica. México: Siglo XXI.

Estudios de Lingüística Aplicada, año 35, número 66, diciembre de 2017, pp. 77-107

doi: 10.22201/enallt.01852647p.2017.66.829 
Ferreiro, Emilia, \& Gómez Palacio, Margarita (1982a). Análisis de las perturbaciones en el proceso de aprendizaje de la lecto-escritura: las relaciones entre el texto — como totalidad-y sus partes. México: Secretaría de Educación Pública, Dirección General de Educación Especial.

Ferreiro, Emilia, \& Gómez Palacio, Margarita (1982b). Análisis de las perturbaciones en el proceso de aprendizaje de la lecto-escritura: las relaciones entre el texto y la imagen. México: Secretaría de Educación Pública, Dirección General de Educación Especial.

Ferreiro, Emilia, \& Teberosky, Ana (1979). Los sistemas de escritura en el desarrollo del niño. México: Siglo XXI.

Figueroa, Verónica, \& Lissi, María Rosa (2005). La lectura en personas sordas: consideraciones sobre el rol del procesamiento fonológico y la utilización del lenguaje de señas. Estudios Pedagógicos, 31(2), 105-119. doi: 10.4067/S0718-07052005000200007

FridMAn Mintz, BorIs (2005). Tense and aspect inflections in Mexican sign language verbs (Tesis doctoral inédita). Georgetown University, Washington, D. C.

Goldin Meadow, Susan, \& Mayberry, Rachel I. (2001). How do profoundly deaf children learn to read? Learning Disabilities Research and Practice, 16(4), 222-229.

Herrera, Valeria; Puente, Aníbal, \& Alvarado, Jesús M. (2014). Visual learning strategies to promote literacy skills in prelingually deaf readers. Revista Mexicana de Psicología, 31(1), 1-10. Recuperado de http://www.redalyc.org/pdf/2430/243033030001.pdf

Herrera, Valeria; Puente, Aníbal; Alvarado, Jesús M., \& Ardila, Alfredo (2007). Códigos de lectura en sordos: la dactilología y otras estrategias visuales y cinéticas. Revista Latinoamericana de Psicología, 39(2), 269-286. Recuperado de http://www.redalyc.org/ pdf/805/80539205.pdf

Hoffmeister, Robert J. (2000). A piece of the puzzle: ASL and reading in deaf children. En Charlene Chamberlain, Jill Patterson Morford \& Rachel I. Mayberry (Eds.), Language acquisition by eye (pp. 143-164). Mahwah: Lawrence Erlbaum.

JANZEN, TERRY (1999). The grammaticalization of topics in American Sign Language. Studies in Language, 23(2), 271-306.

Leybaert, JACQUeline (1998). Phonological representations in deaf children: The importance of early linguistic experience. Scandinavian Journal of Psychology, 39(3), 169-173. Recuperado de http://www.ncbi.nlm.nih.gov/pubmed/9800532

Leybaert, JACQUeline, \& Alegría, Jesús (1995). Spelling development in deaf and hearing children: Evidence for use of morpho-phonological regularities in French. Reading and Writing, $7(1), 89-109$.

Estudios de Lingüística Aplicada, año 35, número 66, diciembre de 2017, pp. 77-107 doi: 10.22201/enallt.01852647p.2017.66.829 
Leybaert, Jacqueline; Content, Alain, \& Alegría, Jesús (2008). The development of written word processing: the case of deaf children. Ilha do Desterro, 21, 1-42. Recuperado de https:// www.researchgate.net/publication/49617737_The_development_of_written_word_processing_the_case_of_deaf_children_The_development_of_written_word_processing_the_case_ of_deaf_children

LidDEll, Scott (1980). American Sign Language syntax. La Haya: De Gruyter Mouton.

Marschark, Marc (1997). Learning to read and write. En Marc Marschark (Ed.), Psychological development of deaf children (pp. 203-227). Nueva York: Oxford University Press.

Massone, María; Buscaglia, Virginia, \& Bogado, Agustina (2010). La comunidad sorda: del trazo a la lengua escrita. Lectura y Vida, 1, 6-17.

Miller, Paul, \& Clark, M. Diane (2011). Phonemic awareness is not necessary to become a skilled deaf reader. Journal of Developmental and Physical Disabilities, 23(5), 459-476.

Morais, José, \& Kolinsky, RÉGIne (1995). The consequences of phonemic awareness. En Beatriz de Gelder \& José Morais (Eds.), Speech and reading: A comparative approach (pp. 317-334). Hove: Lawrence Erlbaum.

Morford, Jill P.; Wilkinson, Erin; Villwock, Agnes; Piñar, Pilar, \& Kroll, Judith F. (2011). When deaf signers read English: Do written words activate their sign translations? Cognition, 118(2), 286-292.

Musselman, Carol (2000). How do children who can't hear learn to read an alphabetic script? A review of the literature on reading and deafness. Journal of Deaf Studies and Deaf Education, 5(1), 9-31.

Niederberger, Nathalie (2007). Apprentissage de la lecture-écriture chez les enfants sourds. Enfance, 59(3), 254-262. Recuperado de https://www.cairn.info/revue-enfance-2007-3page-254.htm

Orduña, Ideitza, \& Ruiz, Luis (2010). Morfemas clasificadores en la Lengua de Señas Mexicana. Signos Lingüísticos, 6(11), 61-84.

Parkes, Malcom (1993). Pause and effect: An introduction to the punctuation in the west. Berkeley: University of California Press.

Paul, Peter V. (2003). Processes and components of reading. En Marc Marschark \& Patricia Elizabeth Spencer (Eds.), Deaf studies, language, and education (pp. 97-109). Nueva York: Oxford University Press.

Pietrosemoli, Lourdes (octubre, 2007). La lectura y la escritura en el sordo: lo que habría que replantear. Conferencia presentada en el xv Encuentro Nacional de Profesionales del Lengua-

Estudios de Lingüística Aplicada, año 35, número 66, diciembre de 2017, pp. 77-107

doi: 10.22201/enallt.01852647p.2017.66.829 
je y la Audición y III Encuentro Internacional Educativo de Profesionales del Lenguaje y la Audición, Salta, Argentina.

Strong, Michael, \& Prinz, Philip (2000). Is American Sign Language skill related to English literacy? En Charlene Chamberlain, Jill Patterson Morford \& Rachel I. Mayberry (Eds.), Language acquisition by eye (pp. 131-141). Mahwah: Lawrence Erlbaum.

Teberosky, Ana (1989). Los conocimientos previos del niño sobre el lenguaje escrito y su incorporación al aprendizaje escolar del ciclo inicial. Revista de Educación, 288, 161-183.

Vernon, Sofía, \& Ferreiro, Emilia (1999). Writing development: A neglected variable in the consideration of phonological awareness. Harvard Educational Review, 69(4), 395-416. 
